\title{
Optimal Grouping and Matching for Network-Coded Cooperative Communications
}

\author{
Sushant Sharma $^{\dagger} \quad$ Yi Shi $^{\star} \quad$ Y. Thomas Hou ${ }^{\star} \quad$ Sastry Kompella ${ }^{\ddagger}$ Scott F. Midkiff ${ }^{\star}$ \\ ${ }^{\dagger}$ Brookhaven National Laboratory, Upton, NY, USA \\ ${ }^{\star}$ Virginia Tech, Blacksburg, VA, USA \\ ${ }_{\ddagger}^{\ddagger}$ U.S. Naval Research Laboratory, Washington D.C., USA
}

\begin{abstract}
Network-coded cooperative communications (NC$\mathrm{CC}$ ) is a new advance in wireless networking that exploits network coding (NC) to improve the performance of cooperative communications (CC). However, there remains very limited understanding of this new hybrid technology, particularly at the link layer and above. This paper fills in this gap by studying a network optimization problem that requires joint optimization of session grouping, relay node grouping, and matching of session/relay groups. After showing that this problem is NPhard, we present a polynomial time heuristic algorithm to this problem. Using simulation results, we show that our algorithm is highly competitive and can produce near-optimal results.
\end{abstract}

\section{KEYWORDS}

Cooperative communications, network coding, grouping, node selection, matching, optimization.

\section{INTRODUCTION}

Recent advance of employing network coding (NC) in cooperative communications (CC) has shown great potential of this new hybrid technology [3], [14], [15], [17], [20], [21], [23]. The so-called NC-CC combines two seemingly orthogonal technologies (CC and $\mathrm{NC}$ ) and exploits $\mathrm{NC}$ to the fullest extent to mitigate potential inefficiency in $\mathrm{CC}$, particularly in a mult-user network. Although some early results have shown the potential of this new hybrid technology, fundamental understanding of NC-CC remains limited, particularly at the link layer and above. The goal of this paper is to fill in this gap by offering some new results on optimal grouping and matching for NC-CC in a multi-user network.

Background. We show how a group of sessions can share a set of relay nodes under NC-CC. Consider a set of $m$ sessions, denoted as $\mathcal{S}=\left\{\left(s_{0}, d_{0}\right),\left(s_{1}, d_{1}\right), \cdots\right.$, $\left.\left(s_{m-1}, d_{m-1}\right)\right\}$ (see Fig. 1(a)) that share the same channel. Under direct transmission (i.e., NC-CC is not employed), a time frame $T$ is divided into $m$ time slots and each session is assigned to a time slot for transmission via TDMA (see Fig. 1(b)). But under NC-CC, a set of relay nodes are employed in data transmission for the $m$ sessions (see Fig. 2). Denote the set of $n$ relay nodes as $\mathcal{R}=\left\{r_{0}, r_{1}, \cdots, r_{n-1}\right\}$. Figure 2(a) shows the time slot structure of a frame under NC-CC. Here, a time frame $T$ is divided into $(m+1)$ time slots. The first $m$ time slots are used by each source node for data transmission (see Figs. 2(b)-(d)). Each of these $m$ transmissions is also overheard by the $n$ relay nodes (in addition to the destination nodes). In the $(m+1)$-th

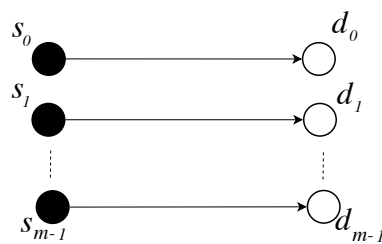

(a) $m$ sources transmit to $m$ destination nodes

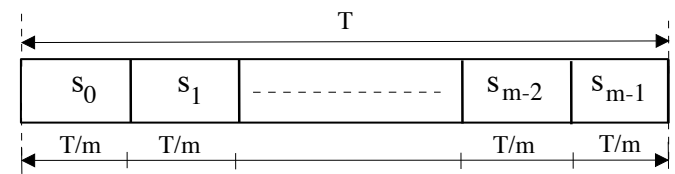

(b) Time slot structure of a frame.

Fig. 1. Direct transmission for a set of communication sessions

time slot, each relay node combines the $m$ received signals via NC (independent from other relay nodes) and broadcast the combined signal in the $(m+1)$-th time slot. Note that these broadcasts by the relay nodes are received by all the destination nodes (see Fig. 2(e)).

At each destination node, some signal processing is needed to extract information from the combined signal in the $(m+$ 1)-th time slot. The details of this extraction process was given in our prior work in [17], where it was shown that for each session $\left(s_{i}, d_{i}\right)$, its mutual information is:

$$
\begin{aligned}
I_{\mathrm{NC}-\mathrm{CC}}\left(s_{i}, \mathcal{S}, \mathcal{R}, d_{i}\right) & =\log _{2}\left(1+\mathrm{SNR}_{s_{i} d_{i}}+\right. \\
& \frac{\left(\sum_{r_{j} \in \mathcal{R}} \sqrt{\frac{\mathrm{SNR}_{r_{j} d_{i}} \mathrm{SNR}_{s_{i} r_{j}}}{|\mathcal{S}|+\sum_{s_{k} \in \mathcal{S}} \mathrm{SNR}_{s_{k} r_{j}}}}\right)^{2}}{\frac{\sigma_{d_{i}}^{2} \mathrm{NC}}{\sigma_{d_{i}}^{2}}+\sum_{r_{j} \in \mathcal{R}}\left(\frac{\mathrm{SNR}_{r_{j} d_{i}}}{|\mathcal{S}|+\sum_{s_{k} \in \mathcal{S}} \mathrm{SNR}_{s_{k} r_{j}}}\right)},
\end{aligned}
$$

where $\sigma_{z_{d_{i}}^{\mathrm{NC}}}^{2}$ is the variance of NC noise at $d_{i}$ (due to NC at the relay nodes), $\mathrm{SNR}_{u v}$ is the signal-to-noise ratio at node $v$ when node $u$ transmits, and is given by $\frac{\left|h_{u v}\right|^{2} P_{u}}{\sigma_{v}^{2}}$. The parameter $h_{u v}$ captures the channel state information (CSI) between nodes $u$ and $v$, and $\sigma_{v}^{2}$ is the variance of white Gaussian background noise at node $v$. The variance of NC noise, $\sigma_{z_{d_{i}}^{\mathrm{NC}}}^{2}$, is given by

$$
\begin{aligned}
\sigma_{z_{i}}^{\mathrm{NC}}=\sigma_{d_{i}}^{2}+|\mathcal{S}-1| \sum_{r_{j} \in \mathcal{R}}\left(\alpha_{r_{j}} h_{r_{j} d_{i}}\right)^{2} \sigma_{r_{j}}^{2}+ \\
\sum_{s_{k} \in \mathcal{S}}^{s_{k} \neq s_{i}} \sum_{r_{j} \in \mathcal{R}}\left(\frac{h_{s_{k} r_{j}} \alpha_{r_{j}} h_{r_{j} d_{i}}}{h_{s_{k} d_{i}}}\right)^{2} \sigma_{d_{i}}^{2},
\end{aligned}
$$




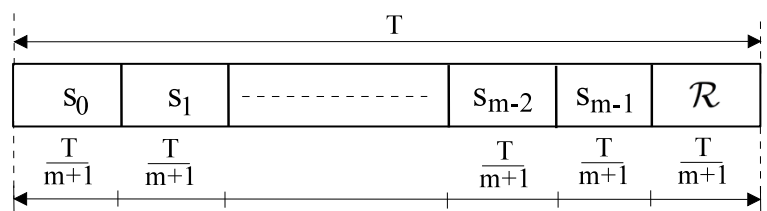

(a) Time slot structure of a frame.

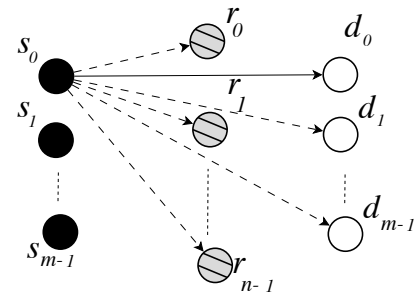

(b) Time slot 1 .

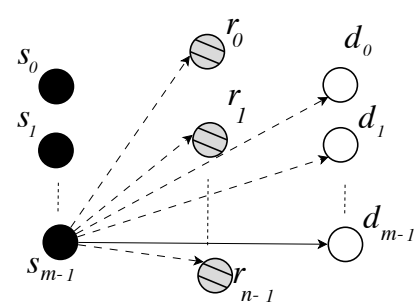

(d) Time slot $m$.

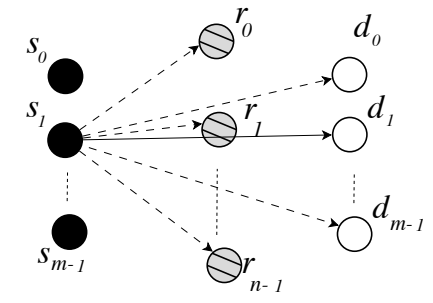

(c) Time slot 2 .

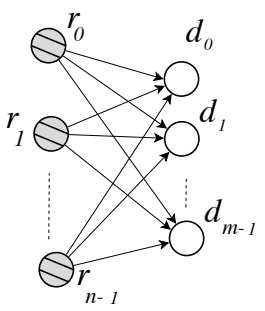

(e) Time slot $m+1$.
Fig. 2. A schematic diagram illustrating the mechanism of NC-CC between a set of sessions and a set of relay nodes.

where $\alpha_{r_{j}}$ is the amplification factor for the relay node $r_{j}$ and is given by

$$
\alpha_{r_{j}}^{2}=\frac{P_{r_{j}}}{\sum_{s_{k} \in \mathcal{S}}\left(\sigma_{r_{j}+}^{2} P_{s_{k}}\left|h_{s_{k} r_{j}}\right|^{2}\right)} .
$$

Finally, the achievable rate for a session $\left(s_{i}, d_{i}\right)$ is

$$
\begin{aligned}
C_{\mathrm{NC}-\mathrm{CC}}\left(s_{i}, \mathcal{S}, \mathcal{R}, d_{i}\right) & =W \cdot \frac{\left(\frac{T}{\mathcal{S} \mid+1}\right)}{T} I_{\mathrm{NC}-\mathrm{CC}}\left(s_{i}, \mathcal{S}, \mathcal{R}, d_{i}\right) \\
& =\frac{W}{|\mathcal{S}|+1} I_{\mathrm{NC}-\mathrm{CC}}\left(s_{i}, \mathcal{S}, \mathcal{R}, d_{i}\right), \quad(3)
\end{aligned}
$$

where $W$ is the channel bandwidth.

Problem Statement. A close look at (1) and (3) suggests that the achievable rate of a session under NC-CC depends on two factors: (i) the number of sessions participating in $\mathrm{NC}-\mathrm{CC}$, and (ii) the channel conditions among the nodes. In a multi-session network, it may not be desirable to put all the sessions in one group and all the relay nodes in another group as in Fig. 2 (as doing so may not optimize the achievable rate of each session). It might be more appropriate to put sessions and relay nodes into different groups and match them up appropriately for optimal performance. This observation leads to the grouping and matching problem that we plan to investigate in this paper.
Our Contributions. In this paper, we investigate the following joint problems in a multi-user network under NCCC: (i) how to put sessions into different groups; (ii) how to put relay nodes into different groups; and (iii) how to match the session groups with relay groups under NC-CC. Specifically, we study a network optimization problem with the goal of maximizing the sum of weighted rates of all sessions. This optimization problem requires a joint optimization of all three components. We show that this problem is NP-hard. Subsequently, we develop a highly competitive and efficient algorithm to solve this problem.

Paper Organization. The rest of the paper is organized as follows. In Section II, we describe the session/relay grouping and matching problem in detail. We also show that this problem is NP-hard. In Section III, we present an algorithm to this problem. Section IV presents numerical results to demonstrate the performance and efficiency of the proposed algorithm. In Section V, we discuss related work, and Section VI concludes this paper.

\section{Problem Description}

Consider a network where there is a set of sessions $\mathcal{S}=\left\{\left(s_{0}, d_{0}\right),\left(s_{1}, d_{1}\right), \cdots,\left(s_{m-1}, d_{m-1}\right)\right\}$ and a set of relay nodes $\mathcal{R}=\left\{r_{0}, r_{1}, \cdots, r_{n-1}\right\}$. For each session $\left(s_{i}, d_{i}\right)$, the source node $s_{i}$ always has data to transmit to the destination node $d_{i}$. We assume that each node can only serve one distinct role of either source, destination, or relay. Assume all the nodes are in the same interference (collision) domain. Therefore, similar to Fig. 2(a), a time frame of length $T$ needs to be divided among the sessions to coordinate transmissions. Given the availability of relay nodes, NC-CC may be used. Our goal is to exploit the potential of NC-CC and set up a transmission schedule so that our performance objective is optimized.

In this network setting, a number of questions arise naturally.

- First and foremost, from each session's perspective, what set of relay nodes should it employ to increase its achievable rate?

- Second, from each relay node's perspective, what set of sessions should it support (in the context of NC-CC)?

- Third, should we partition the set of sessions and relay nodes into different groups? And if so, how to group these sessions and relay nodes, and how to match them to optimize our objective?

- Finally, how should the time slots in a frame be structured so as to coordinate the transmissions of all sessions?

Regarding the first question, one can quickly deduce, by a simple numerical analysis of (1), that blind employment of all relay nodes in the network may not maximize a session's 
achievable rate. This is because background noise, introduced in the received signals at certain relay nodes, could be high. Once such noisy signal is amplified, transmitted, and aggregated with signals from other relay nodes, it will lead to large noise in the received signal at a destination node, thereby reducing the session's achievable rate. Another issue is that the variance of NC noise increases monotonically with the size of the relay group. Therefore, in the interest of each session, it is important to select an optimal subset of relay nodes to maximize its achievable rate.

For the second question, by observing (2), one can easily find that the variance of $\mathrm{NC}$ noise increases monotonically as the number of sessions. Since the achievable rate decreases as NC noise variance increases, we conclude that loading a relay node with a large number of sessions will not maximize our objective. Therefore, from a relay node's perspective, it is important to select an optimal subset of sessions.

Based on the above discussion, it is easy to answer the third question. Clearly, we need to partition the set of sessions and relay nodes into different groups. Note that there could be some overlap among the sets of relay nodes, i.e., a relay node may lie in multiple groups. However, a session can only appear in one group. As we shall show, grouping of sessions and relay nodes is not a trivial task, neither is the problem of matching them to maximize our objective.

For the last question, once the optimal session/relay grouping and matching problem is solved, the time slot structure can be determined using a simple scheme as follows. A set $\mathcal{S}_{k}$ with $\left|\mathcal{S}_{k}\right|$ sessions will have total available time of $\left|\mathcal{S}_{k}\right| t$. When this session group uses a relay group $\mathcal{R}_{j}$ for NC-CC, the time slot duration for every session in $\mathcal{S}_{k}$ will shrink to $\frac{\left|\mathcal{S}_{k}\right| t}{\left|\mathcal{S}_{k}\right|+1}$. As a result, the achievable rate for a session $\left(s_{i}, d_{i}\right)$ is

$$
\begin{aligned}
& C_{\mathrm{NC}-\mathrm{CC}}\left(s_{i}, \mathcal{S}_{s_{i}}^{\mathcal{R}_{j}}, \mathcal{R}_{j}, d_{i}\right) \\
= & W \cdot \frac{\left(\frac{\left|\mathcal{S}_{s_{i}}^{\mathcal{R}_{j}}\right| t}{\left|\mathcal{S}_{s_{i}}\right|+1}\right)}{|\mathcal{S}| t} \cdot I_{\mathrm{NC}-\mathrm{CC}}\left(s_{i}, \mathcal{S}_{s_{i}}^{\mathcal{R}_{j}}, \mathcal{R}_{j}, d_{i}\right) \\
= & \frac{\left|\mathcal{S}_{s_{i}}^{\mathcal{R}_{j}}\right|}{\left|\mathcal{S}_{s_{i}} \mathcal{R}_{j}\right|+1} \cdot \frac{W}{|\mathcal{S}|} \cdot I_{\mathrm{NC}-\mathrm{CC}}\left(s_{i}, \mathcal{S}_{s_{i}}^{\mathcal{R}_{j}}, \mathcal{R}_{j}, d_{i}\right), \\
& \mathcal{S}_{s_{i}}^{\mathcal{R}_{j}} \subseteq \mathcal{S}, s_{i} \in \mathcal{S}_{s_{i}}^{\mathcal{R}_{j}},
\end{aligned}
$$

where $\mathcal{S}_{s_{i}}^{\mathcal{R}_{j}}$ denotes the session group (containing session $\left.\left(s_{i}, d_{i}\right)\right)$ that is matched to relay nodes in $\mathcal{R}_{j}$.

Problem Complexity. Our goal in this paper is to perform optimal grouping of sessions and relay nodes, and matching these groups so that the sum of weighted session rate is maximized. For session grouping, the smaller the size of each group, the larger the mutual information, due to smaller NC noise. But on the other hand, comparing (3) to (5), we find that smaller session group size will also have smaller effective bandwidth (i.e., $\frac{\left|\mathcal{S}_{i}^{\mathcal{R}_{j}}\right|}{\left|\mathcal{S}_{s_{i}}{ }^{\mathcal{R}_{j}}\right|+1} \frac{W}{|\mathcal{S}|}<\frac{W}{|\mathcal{S}|+1}$ because $|\mathcal{S}|$ is greater than $\left|\mathcal{S}_{s_{i}}^{\mathcal{R}_{j}}\right|$ ). For grouping of relay nodes, there is even more flexibility, as any relay node may be part of multiple relay groups. Finally, the optimal matching problem is highly complex, due to the large design space of potential session groups and relay groups.

Theorem 1: The joint session/relay grouping and matching problem for NC-CC is NP-hard.

We give a sketch of proof as follows. In [16], Sharma et al. considered a simpler grouping and relay node selection (GRS) problem, with the same objective of maximizing the weighted sum rate of the sessions in the network. There was no consideration of grouping of relay nodes. In other words, the size of each relay group was set to 1, which can be viewed as a special case of the problem in this paper. For the GRS problem, Sharma et al. used matching problems in hypergraphs to show that the GRS problem is NP-hard. Given that the GRS problem is a special case of our joint session/relay grouping and matching problem, we can conclude that our problem is at least NP-hard.

\section{G ${ }^{2}$ M: AN Algorithm FOR Session/RELAY GROUPING AND MATCHING}

In this section, we present an algorithm that performs session grouping, relay grouping, and matching of session and relay groups. We abbreviate this algorithm as $\mathrm{G}^{2} \mathrm{M}$, with the " 2 " referring that grouping operation is performed on both sessions and relay nodes. We present the $\mathrm{G}^{2} \mathrm{M}$ algorithm in Sections III-A and III-B.

\section{A. Basic Idea}

The basic idea of $\mathrm{G}^{2} \mathrm{M}$ is to have each session initially matched independently to a group of relay nodes. That is, we try to maximize the mutual information for each session independently. Then through merging of sessions and modifications of relay node groups iteratively, we obtain a final solution.

In the initialization phase, we let each session $\left(s_{i}, d_{i}\right), i=$ $0, \cdots, m-1$, form a group on its own, i.e., $\mathcal{S}_{i}=\left\{\left(s_{i}, d_{i}\right)\right\}$, $i=0, \cdots, m-1$. Then for each session group $\mathcal{S}_{i}$ (which has only one session), we find a set of relay nodes for it, which we denote as $\mathcal{R}_{i}, i=0, \cdots, m-1$. The set of relay nodes is determined through an iterative process that begins by considering all the relay nodes in the set, and then removing some relay nodes from the set that are not helpful to that particular session.

In the main program, during each iteration, we consider pair-wise of session groups and see if merging the two will result in an improved objective function. Clearly, merging of two session groups also requires the merging of two groups of relay nodes. To increase the chance of successful merger 
of two session groups, modifications of relay nodes (in terms of removing some nodes) are allowed in the newly merged relay node groups. Such iteration terminates when we can no longer find a pair of session groups to merge that can produce a greater objective value. At this point, $G^{2} \mathrm{M}$ terminates.

\section{B. Algorithm Details}

Initialization. As discussed in Section III-A, we start by having each session group contain only one session, i.e. $\mathcal{S}_{i}=$ $\left\{\left(s_{i}, d_{i}\right)\right\}, i=0, \cdots, m-1$. For each session $\mathcal{S}_{i}$, we will find a group of relay nodes $\mathcal{R}_{i}, i=0, \cdots, m-1$ for it so that the achievable rate of this session is maximized. Based on (1), when $\mathcal{S}_{i}=\left\{\left(s_{i}, d_{i}\right)\right\}$ is matched to a relay group $\mathcal{R}_{i}$, its mutual information is

$$
\begin{aligned}
I_{\mathrm{NC}-\mathrm{CC}}\left(s_{i},\left\{\left(s_{i}, d_{i}\right)\right\}, \mathcal{R}_{i}, d_{i}\right)=\log _{2}\left(1+\mathrm{SNR}_{s_{i} d_{i}}\right. \\
\left.+\frac{\left(\sum_{r_{j} \in \mathcal{R}_{i}} \sqrt{\left.\frac{\mathrm{SNR}_{r_{j} d_{i}} \mathrm{SNR}_{s_{i} r_{j}}}{1+\mathrm{SNR}_{s_{i} r_{j}}}\right)^{2}}\right.}{1+\sum_{r_{j} \in \mathcal{R}_{i}}\left(\frac{\mathrm{SNR}_{r_{j} d_{i}}}{1+\mathrm{SNR}_{s_{i} r_{j}}}\right)}\right) .
\end{aligned}
$$

From (6), the SNR-gain for $\left(s_{i}, d_{i}\right)$ due to the relay group $\mathcal{R}_{i}$ is

$$
\operatorname{SNR}_{\text {gain }}\left(s_{i}, \mathcal{R}_{i}\right)=\frac{\left(\sum_{r_{j} \in \mathcal{R}_{i}} \sqrt{\frac{\mathrm{SNR}_{r_{j} d_{i}} \mathrm{SNR}_{s_{i} r_{j}}}{1+\mathrm{SNR}_{s_{i} r_{j}}}}\right)^{2}}{1+\sum_{r_{j} \in \mathcal{R}_{i}}\left(\frac{\mathrm{SNR}_{r_{j} d_{i}}}{1+\mathrm{SNR}_{s_{i} r_{j}}}\right)} .
$$

Now we need to find a group of relay nodes $\mathcal{R}_{i}$ for each $\mathcal{S}_{i}$ that can maximize $\mathrm{SNR}_{\text {gain }}$. The following theorem shows that this problem is also NP-hard.

Theorem 2: For a single session, the problem of finding an optimal group of relay nodes that maximizes the session's achievable rate is NP-hard.

We offer a sketch of proof here. In [16], Sharma et al. showed that the problem of having a single relay node to select an optimal group of sessions among a set of sessions is NP-hard. The proof technique there was based on matching problems in hypergraphs. The mathematical nature of that problem is exactly the same as this one, and thus the proof here can follow the same token.

We now present a heuristic algorithm to construct an initial matching.

- For $\mathcal{S}_{i}=\left(s_{i}, d_{i}\right)$, we start with a group $\mathcal{R}_{i}$ that includes all the relay nodes in the network.

- To maximize $\mathrm{SNR}_{\text {gain }}$, we identify and remove certain relay nodes (one at a time) from $\mathcal{R}_{i}$.

- The first candidate for possible removal is the relay node with the poorest channel condition between $s_{i}$ and itself, i.e., the relay node $r_{j}$ with the smallest value of $\mathrm{SNR}_{s_{i} r_{j}}$. This node is likely to introduce the largest noise component. From (6), we can see that this relay node is also likely to contribute the largest amount to the denominator.

- Remove this relay node, say $r_{j}$ with the smallest $\mathrm{SNR}_{s_{i} r_{j}}$. If session $\left(s_{i}, d_{i}\right)$ 's mutual information increases, this removal is permanent; otherwise, $r_{j}$ is added back to $\mathcal{R}_{i}{ }^{1}$

- Repeat the above process for the relay node with the second smallest value of $\mathrm{SNR}_{s_{i} r_{j}}$ and so forth.

- During the above iteration for $\mathcal{S}_{i}$, some relay nodes may be removed from $\mathcal{R}_{i}$. As a result, we should go through another iteration of checking and removing the relay nodes from the current $\mathcal{R}_{i}$. This is due to the nonlinear nature of (7). Note that in the above iteration, when we check the current relay nodes in $\mathcal{R}_{i}$ for removal, the relay nodes in $\mathcal{R}_{i}$ at that time are different from the current $\mathcal{R}_{i}$. Thus, it may now be possible to further remove some of the current relay nodes and improve $\mathcal{R}_{i}$.

- We terminate the process of removing the relay nodes from $\mathcal{R}_{i}$ until none of the remaining relay nodes can be removed from $\mathcal{R}_{i}$. The current set of relay nodes in $\mathcal{R}_{i}$ constitutes the initial group of relay nodes that is matched to $\mathcal{S}_{i}=\left(s_{i}, d_{i}\right)$.

- As a last step, we want to ensure that the achievable rate of session $\left(s_{i}, d_{i}\right)$ is no less than that under direct transmission. If yes, we will keep this relay group; otherwise, we set $\mathcal{R}_{i}=\emptyset$, indicating that initially no relay node will be matched to this session.

Main Program. After initialization, we have an initial list (say $\mathcal{L}_{1}$ ) of $m=|\mathcal{S}|$ matchings with every session group (containing a single session) matched to a group of relay nodes. Note that NC is not yet employed and the goal of the main program is to merge session groups (two at a time) so that $\mathrm{NC}$ can be fully exploited to increase the objective of our optimization problem (i.e., weighted sum rate of all sessions)

In the first iteration, we go through the initial list $\mathcal{L}_{1}$ that has $m$ entries of $\left(\mathcal{S}_{i}, \mathcal{R}_{i}\right)$ matchings. We consider all possible pairs of entries $\left(\mathcal{S}_{i}, \mathcal{R}_{i}\right)$ and $\left(\mathcal{S}_{j}, \mathcal{R}_{j}\right), \mathcal{S}_{i} \neq \mathcal{S}_{j}$, for possible merger. There are $\frac{m(m-1)}{2}$ possibilities.

Denote $\mathcal{L}_{\text {temp }}$ a temporary working list to store our intermediate matching results. For every matching pair of entries $\left[\left(\mathcal{S}_{i}, \mathcal{R}_{i}\right),\left(\mathcal{S}_{j}, \mathcal{R}_{j}\right)\right]$ in $\mathcal{L}_{1}$, we perform the following steps.

- Suppose two session groups $\mathcal{S}_{i}$ and $\mathcal{S}_{j}$ are merged into one session group $\mathcal{S}_{i} \cup \mathcal{S}_{j}$. Then the two corresponding relay node groups $\mathcal{R}_{i}$ and $\mathcal{R}_{j}$ are also merged into one

\footnotetext{
${ }^{1}$ This is because that, from (7), not only the values of $\mathrm{SNR}_{s_{i} r_{j}}$, but also the values of $\mathrm{SNR}_{r_{j} d_{i}}$ and the $\mathrm{SNR}$ values of the other relay nodes are affecting the value of $\mathrm{SNR}_{\text {gain }}$.
} 
relay node group $\mathcal{R}_{i} \cup \mathcal{R}_{j}$. Now we have a new session group $\mathcal{S}_{i} \cup \mathcal{S}_{j}$ matched to a new relay group $\mathcal{R}_{i} \cup \mathcal{R}_{j}$.

- Given that $\mathcal{R}_{i}$ and $\mathcal{R}_{j}$ likely contain different sets of nodes, some of which may benefit sessions in one group but not the other. To ensure that every relay node in $\mathcal{R}_{i} \cup \mathcal{R}_{j}$ will benefit the new session group $\mathcal{S}_{i} \cup \mathcal{S}_{j}$, we examine each non-overlapping relay node in $\mathcal{R}_{i} \cup \mathcal{R}_{j}$ (i.e., the relay nodes that are not part of both $\mathcal{R}_{i}$ and $\mathcal{R}_{j}$ ) one at a time and remove any relay, say $r_{k}$ if its presence in $\mathcal{R}_{i} \cup \mathcal{R}_{j}$ is harmful to the objective function for the new session group $\mathcal{S}_{i} \cup \mathcal{S}_{j}$. After this process, we have an updated relay group, which we denote as $\left(\mathcal{R}_{i} \cup \mathcal{R}_{j}\right)^{*}$.

- To determine whether or not the proposed new matching $\left(\mathcal{S}_{i} \cup \mathcal{S}_{j},\left(\mathcal{R}_{i} \cup \mathcal{R}_{j}\right)^{*}\right)$ should be stored in $\mathcal{L}_{\text {temp }}$, we compare whether or not there is any improvement in the objective function, i.e., whether or not

$\sum_{s_{k} \in \mathcal{S}_{i} \cup \mathcal{S}_{j}} w_{k} C_{\mathrm{NC}-\mathrm{CC}}\left(s_{k}, \mathcal{S}_{i} \cup \mathcal{S}_{j},\left(\mathcal{R}_{i} \cup \mathcal{R}_{j}\right)^{*}, d_{k}\right)>$ $\left(\sum_{s_{k} \in \mathcal{S}_{i}} w_{k} C_{\mathrm{NC}-\mathrm{CC}}\left(s_{k}, \mathcal{S}_{i}, \mathcal{R}_{i}, d_{k}\right)+\right.$ $\left.\sum_{s_{k} \in \mathcal{S}_{j}} w_{k} C_{\mathrm{NC}-\mathrm{CC}}\left(s_{k}, \mathcal{S}_{j}, \mathcal{R}_{j}, d_{k}\right)\right)$ ?

- If there is an increase in objective, then we store the new matching $\left(\mathcal{S}_{i} \cup \mathcal{S}_{j},\left(\mathcal{R}_{i} \cup \mathcal{R}_{j}\right)^{*}\right)$ in $\mathcal{L}_{\text {temp }}$. Also, we calculate the net increase in the objective value due to this merger, which we call temporary gain.

- If the objective value decreases or remains same, then there is no benefit in merging $\mathcal{S}_{i}$ and $\mathcal{S}_{j}$. Therefore, we declare this proposed merger a failure. If $\left(\mathcal{S}_{i}, \mathcal{R}_{i}\right)$ and $\left(\mathcal{S}_{j}, \mathcal{R}_{j}\right)$ have not been stored in $\mathcal{L}_{\text {temp }}$, we will store both as two entries in $\mathcal{L}_{\text {temp }}$ and associate each with a zero temporary gain.

We now have a list $\mathcal{L}_{\text {temp }}$ containing several beneficial matchings and some matchings with zero gain. Note that a session group $\mathcal{S}_{i}$ may be part of multiple matchings in list $\mathcal{L}_{\text {temp }}$. We now want to create a list $\mathcal{L}_{2}$ where any session group $\mathcal{S}_{i}$ will only appear in exactly one matching. This is equivalent to having each session appear only once in some session group in $\mathcal{L}_{2}$. To accomplish this, we consider entries in $\mathcal{L}_{\text {temp }}$ in decreasing value of temporary gain. For any such entry under consideration, we do the following.

- If none of the sessions in this session group appears in any session group of $\mathcal{L}_{2}$, this entry of matching (session group and relay node group) is saved in $\mathcal{L}_{2}$. This entry is also removed from $\mathcal{L}_{\text {temp }}$.

- If all sessions in this session group already appear in some session groups in $\mathcal{L}_{2}$, this entry is not saved in $\mathcal{L}_{2}$. Further, this entry is also removed from $\mathcal{L}_{\text {temp }}$.

- If some, but not all, sessions of this session group appear in some session groups in $\mathcal{L}_{2}$, then we will recover the session group containing the remaining sessions (i.e., those not showing up in $\mathcal{L}_{2}$ ) and its matching relay group from $\mathcal{L}_{1}$. This recovered matching entry will carry a temporary gain of zero and will replace the one in $\mathcal{L}_{\text {temp }}$.

The above process continues until $\mathcal{L}_{\text {temp }}$ is empty. At this point, each session should appear only once in some session group in $\mathcal{L}_{2}$. This completes the first iteration of our main program.

Future iterations of the main program are similar to the first iteration. The program terminates when no further mergers are possible, i.e., the temporary gain is zero for all entries in $\mathcal{L}_{\text {temp. }}$. Then the matching created in the previous iteration is our final solution. The total complexity of $\mathrm{G}^{2} \mathrm{M}$ is $O\left(|\mathcal{S}|^{2}|\mathcal{R}|^{2}+|\mathcal{S}|^{3}|\mathcal{R}|+|\mathcal{S}|^{5}\right)$

\section{NumericAl RESUlts}

In this section, we present numerical results to demonstrate the performance and efficiency of our $\mathrm{G}^{2} \mathrm{M}$ algorithm. Our goals are twofold: (i) to show $\mathrm{G}^{2} \mathrm{M}$ algorithm offers better results than direct transmission, and (ii) to demonstrate that the solutions constructed by $\mathrm{G}^{2} \mathrm{M}$ are close to the optimal solutions obtained by CPLEX solver [5].

\section{A. Parameter Settings}

For all network instances used in this simulation study, we assume the transmission power at each node is $1 \mathrm{~W}$. The available transmission bandwidth at every node is $20 \mathrm{MHz}$, and the variance of white Gaussian background noise at all nodes is $10^{-10} \mathrm{~W}$. The channel gain between two nodes $s$ and $d$ is modeled as $\left|h_{s d}\right|^{2}=\|s-d\|^{-4}$, where $\|s-d\|$ is the distance between $s$ and $d$ (in meters).

\section{B. Results}

1) $G^{2} M$ vs. Direct Transmission: We consider 100 randomly generated network instances, each with 30 nodes (7 source-destination pairs and 16 relay nodes). For each instance, the nodes are randomly deployed in a $1200 \mathrm{~m} \mathrm{x}$ $1200 \mathrm{~m}$ square area. We calculate the objective value for each network instance under both $\mathrm{G}^{2} \mathrm{M}$ and direct transmission. Figure 3(a) plots the ratio of the objective values obtained under $\mathrm{G}^{2} \mathrm{M}$ and those under direct transmission when all the weights in the network are set to 1. Similarly, Fig. 3(b) plots the ratio when each session's weight in the network is randomly chosen between 0 and 1. In Fig. 3(a), the average ratio is 2.53 (with a variance of 2.83); in Fig. 3(b), the average ratio is 2.67 (with a variance of 3.98 ). Note that under any network instance in each figure, the ratio between the two is always greater than 1 .

2) Near-Optimality of $G^{2} M$ : To validate the performance of $\mathrm{G}^{2} \mathrm{M}$, we compare the results by $\mathrm{G}^{2} \mathrm{M}$ to the optimal solutions obtained by solving a mathematical formulation of our session/relay grouping and matching problem. A 


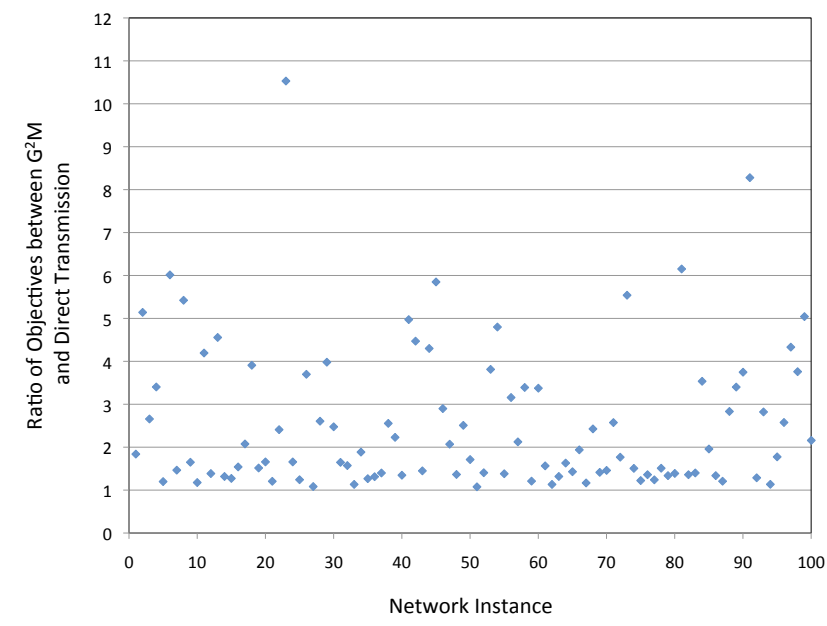

(a) $w_{i}=1$ for $i=0, \cdots,|\mathcal{S}|-1$.

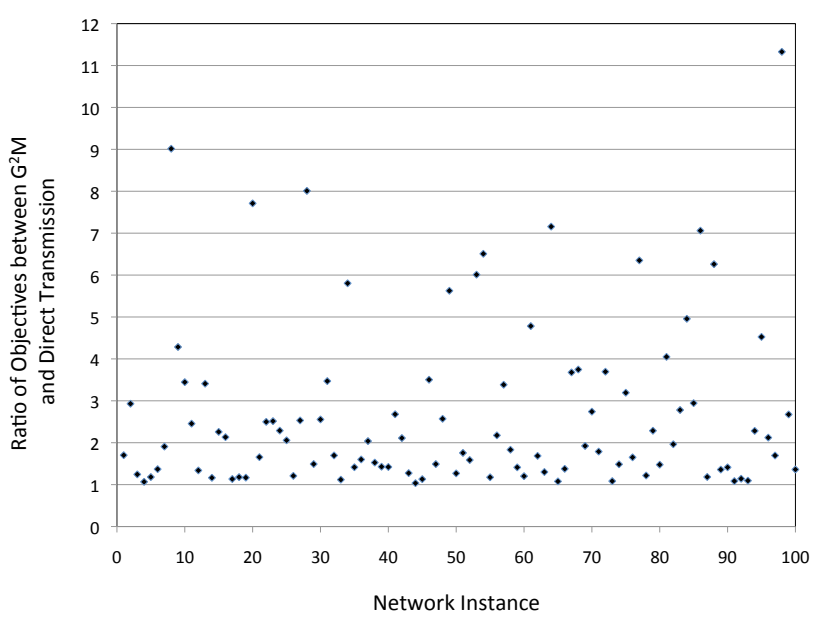

(b) Each $w_{i}$ is randomly generated between $[0,1] ; i=0, \cdots,|\mathcal{S}|-1$.

Fig. 3. Ratios between the objective values under $G^{2} M$ and Direct Transmission.

mathematical formulation of this problem is in the form of 0-1 integer linear program (ILP), and is omitted due to page limitation.

Figure 4(a) shows the ratio between the objective values obtained by $\mathrm{G}^{2} \mathrm{M}$ over those from CPLEX when the weight of each session is set to 1 . Similarly, Fig. 4(b) shows the ratio between the two when the weight of each session is randomly set between $[0,1]$. As we can see, the performance of $\mathrm{G}^{2} \mathrm{M}$ is highly competitive in both cases. It is $98.8 \%$ of optimal on average (with a variance of 0.05) for fixed weights, and $97.7 \%$ optimal on average (with a variance of 0.15 ) for random weights. Note that the runtime complexity of the centralized solver that solves ILP is exponential (as opposed to polynomial for the $\mathrm{G}^{2} \mathrm{M}$ algorithm).

\section{RELATED WORK}

Although $\mathrm{CC}$ has been an active research area for many years (see, e.g., [1], [4], [6], [7], [9], [8], [10], [11], [12],

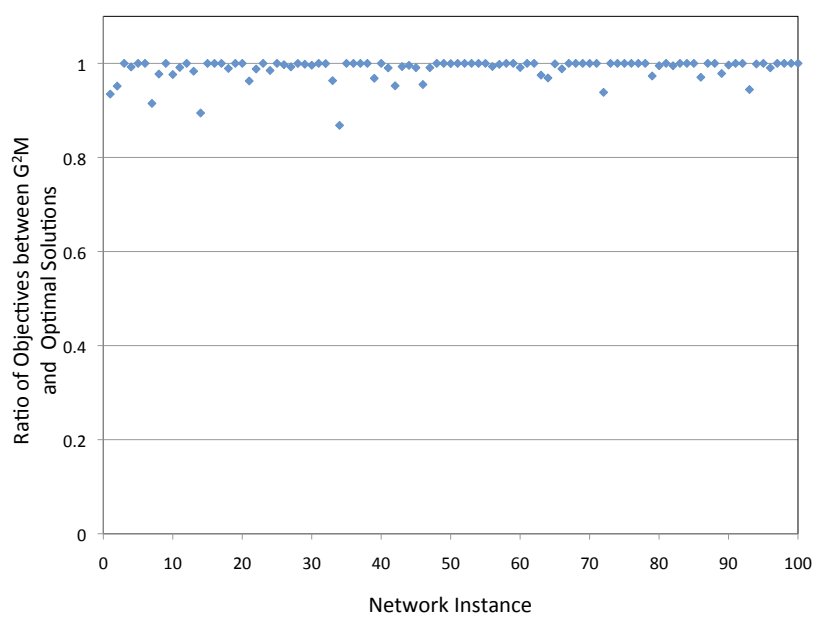

(a) $w_{i}=1$ for $i=0, \cdots,|\mathcal{S}|-1$.

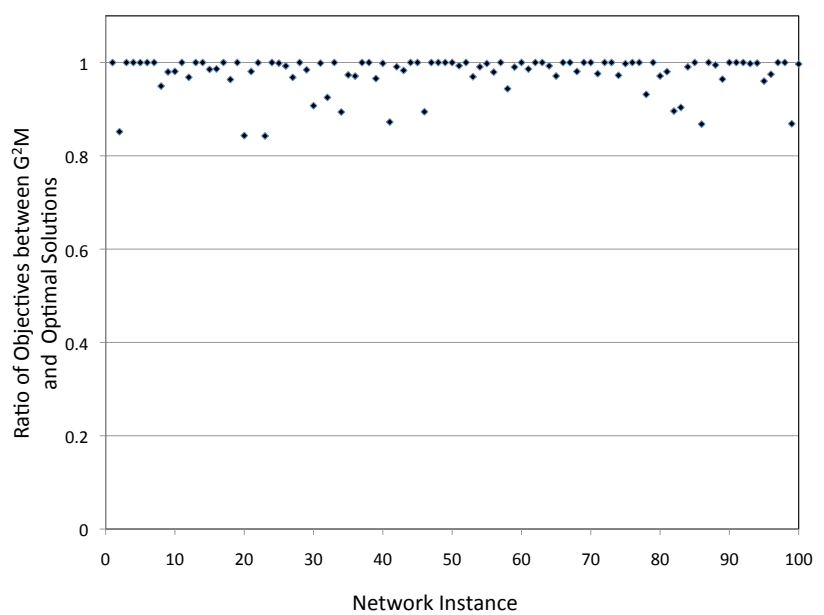

(b) Each $w_{i}$ is randomly generated between $[0,1] ; i=0, \cdots,|\mathcal{S}|-1$.

Fig. 4. Ratios between the objective values under $\mathrm{G}^{2} \mathrm{M}$ and CPLEX.

[19], [18], [22]), recent development in employing NC in CC (so-called NC-CC) has chartered a new research direction. To date, research on $\mathrm{NC}-\mathrm{CC}$ is still in its early stage and results remain limited [3], [14], [15], [20], [21], [23]. In [3], Bao and Li were the first to employ NC-CC in a multisource single-destination network. Their focus was to develop coding mechanisms that could be used by the source nodes to cooperate with each other. In [14], Peng et al. considered a network with a single relay node and multiple sourcedestination pairs, and studied the outage probability of the entire network when NC-CC is employed. Sharma et al. [15] also considered a network with a single relay node and multiple source-destination pairs, and derived achievable rate for individual session under NC-CC. Xiao et al. [20] considered a two-source single-destination network and showed that NC can help CC reduce packet error rates. In [21] and [23], the NC-CC framework was limited in exploiting NC only in case of bi-directional traffic and by using a single relay node. We have shown in this paper that NC-CC is beneficial in 
unidirectional traffic as well, and multiple relay $\mathrm{NC}-\mathrm{CC}$ can be significantly better than single relay $\mathrm{NC}-\mathrm{CC}$. As a result, limiting the work to bi-directional traffic only and the use of a single relay node limits the potential gains of their approach in an ad-hoc network. In fact, a common limitation of all these prior efforts is the use of only a single relay node. As a result, they could not benefit from any performance gains that can be offered by multiple relay nodes. NC-CC with multiple relay nodes was first explored by Sharma et al. in [17], where they showed that a proper choice of a group of relay nodes could have a significant impact on NC-CC's performance. However, the problem on how to group sessions, relay nodes, and match them together remains open. This paper is the first attempt to address this important problem.

\section{CONCLUSIONS}

$\mathrm{NC}-\mathrm{CC}$ is a new research direction in CC. As a result, fundamental results in this area remain lacking, particularly at the link layer and above. The goal of this paper was to fill in this gap by offering new results on NC-CC. By studying a network optimization problem, we developed new understanding of NC-CC in multiple dimensions such as session grouping, relay node grouping, and matching of session/relay groups. We presented a polynomial time heuristic algorithm to this problem. Using simulation results, we showed that the proposed algorithm is highly competitive and can offer near-optimal results.

\section{ACKNOWLEDGMENTS}

The work of Y.T. Hou, S.F. Midkiff, S. Sharma, and Y. Shi was supported in part by NSF under Grant CNS-1064953. The work of S. Kompella was supported in part by the ONR.

\section{REFERENCES}

[1] B. Aazhang, R.S. Blum, J.N. Laneman, K.J.R. Liu, W. Su, and A. Wittneben (editors), IEEE Journal on Selected Areas in Communications - Special Issue on Cooperative Communications and Networking, vol. 25, no. 2, Feb. 2007.

[2] R. Ahlswede, N. Cai, S-Y.R. Li, and R.W. Yeung, "Network information flow," IEEE Transactions on Information Theory, vol. 46, issue 4, pp. 1204-1216, August 2002.

[3] X. Bao and J. Li, "Adaptive network coded cooperation (ANCC) for wireless relay networks: Matching code-on-graph with network-on-graph," IEEE Transactions on Wireless Communications, vol. 7, no. 2, pp. 574-583, February 2008.

[4] T.M. Cover and A. EL Gamal, "Capacity theorems for the relay channel," IEEE Transactions on Information Theory, vol. 25 , issue 5, pp. 572-584, 1979.

[5] IBM ILOG CPLEX: http://www.ibm.com/software/integration/optimization/cplex-optimizer/.

[6] G. Jakllari, S.V. Krishnamurthy, M. Faloutsos, P.V. Krishnamurthy, and O. Ercetin, "A cross-layer framework for exploiting virtual MISO links in mobile ad hoc networks," IEEE Transactions on Mobile Computing, vol. 6, no. 5, pp. 579-594, June 2007.
[7] G. Jakllari, S.V. Krishnamurthy, M. Faloutsos, and P.V. Krishnamurthy, "On broadcasting with cooperative diversity in multi-hop wireless networks," IEEE Journal on Selected Areas in Communications, vol. 25, no. 2, pp. 484-496, February 2007.

[8] G. Kramer, I. Maric, and R.D. Yates, Foundations and Trends in Networking: Cooperative Communications, NOW publishers, vol. 1, nos. 3-4, 2006.

[9] G. Kramer, R. Berry, A. El Gamal, H. El Gamal, M. Franceschetti, M. Gastpar, and J.N. Laneman (editors), IEEE Transactions on Information Theory - Special Issue on Models, Theory, and Codes for Relaying and Cooperation in Communication Networks, vol. 53, no. 10, Oct. 2007.

[10] S. Lakshmanan and R. Sivakumar, "Diversity routing for multi-hop wireless networks with cooperative transmissions," in Proc. IEEE SECON, Rome, Italy, June 22-26, 2009.

[11] J.N. Laneman, D.N.C. Tse, and G.W. Wornell, "Cooperative diversity in wireless networks: Efficient protocols and outage behavior," IEEE Transactions on Information Theory, vol. 50, no. 12, pp. 3062-3080, Dec. 2004.

[12] K.J.R. Liu, A.K. Sadek, W. Su, and A. Kwasinski, Cooperative Communications and Networking, Cambridge University Press, 2009.

[13] Network Coding Bibliography: https://hermes.lnt.e-technik.tumuenchen.de/DokuWiki/doku.php?id=network_coding: bibliography_for_network_coding.

[14] C. Peng, Q. Zhang, M. Zhao, and Y. Yao, "On the performance analysis of network-coded cooperation in wireless networks," in Proc. IEEE INFOCOM, pp. 1460-1468, Anchorage, Alaska, May 6-12, 2007.

[15] S. Sharma, Y. Shi, J. Liu, Y.T. Hou, and S. Kompella, "Is network coding always good for cooperative communciations," in Proc. IEEE INFOCOM, San Diego, CA, March 15-19, 2010.

[16] S. Sharma, Y. Shi, Y.T. Hou, H.D. Sherali, and S. Kompella, "Optimizing network-coded cooperative communications via joint session grouping and relay node selection," in Proc. IEEE INFOCOM, Shanghai, China, April 10-15, 2011.

[17] S. Sharma, Y. Shi, Y.T. Hou, and S. Kompella, "Achievable rate analysis in network-coded cooperative communications with multiple relay nodes," in Proc. IEEE ICC - Wireless Networking Symposium, Kyoto, Japan, June 5-9, 2011.

[18] S. Sharma, Y. Shi, Y.T. Hou, and S. Kompella, "An optimal algorithm for relay node assignment in cooperative ad hoc networks," IEEE/ACM Transactions on Networking, vol. 19, issue 3, pp. 879-892, June 2011.

[19] E.C. van der Meulen, "Three terminal communication channels," Advances in Applied Probability, vol. 3, pp. 120-154, 1971.

[20] L. Xiao, T.E. Fuja, J. Kliewer, and D.J. Costello, "A network coding approach to cooperative diversity," IEEE Transactions on Information Theory, vol. 53, no. 10, pp. 3714-3722, Oct. 2007.

[21] H. $\mathrm{Xu}$ and B. Li, "XOR-assisted cooperative diversity in OFDMA wireless networks: Optimization framework and approximation algorithms," in Proc. IEEE INFOCOM, pp. 21412149, Rio de Janeiro, Brazil, April 19-25, 2009.

[22] E.M. Yeh and R.A. Berry, "Throughput optimal control of cooperative relay networks," IEEE Transactions on Information Theory, vol. 53, no. 10, pp. 3827-3833, Oct. 2007.

[23] J. Zhang and Q. Zhang, "Cooperative network coding-aware routing for multi-rate wireless networks," in Proc. IEEE INFOCOM, pp. 181-189, Rio de Janeiro, April 19-25, 2009. 